\title{
Herpes Simplex Encephalitis as a Complication of Whole-Brain Radiotherapy: A Case Report and Review of the Literature
}

\author{
David J. Sermer ${ }^{a, b}$ Jamie L. Woodley ${ }^{c}$ Christian A. Thomas ${ }^{d}$ \\ Jacquelyn A. Hedlund ${ }^{d}$ \\ ${ }^{a}$ Duke University Medical Center, Durham, N.C., ${ }^{b}$ Tufts University School of Medicine, \\ Boston, Mass., 'College of Osteopathic Medicine, University of New England, \\ Biddeford, Me., and ${ }^{d}$ New England Cancer Specialists, Scarborough, Me., USA
}

\section{Key Words}

Herpes simplex encephalitis · Cranial irradiation · Brain metastasis · Lung adenocarcinoma · Immunosuppression

\begin{abstract}
A 55-year-old male recently diagnosed with stage IV lung adenocarcinoma presented with altered mental status approximately 1 week after the completion of 14 fractions of wholebrain radiotherapy (WBRT) for brain metastases. On admission, he was somnolent but oriented and without focal neurological deficits. Brain imaging revealed marked regression of his brain metastases. Laboratory values were only significant for hyponatremia with urine hyperosmolality consistent with syndrome of inappropriate antidiuretic hormone secretion. The patient developed seizures 3 days after admission, at which time cerebrospinal fluid was significant for positive herpes simplex virus (HSV)-1 PCR but with a negative cell count, and acyclovir was started for HSV encephalitis (HSE). After 3 weeks of acyclovir $10 \mathrm{mg} / \mathrm{dl}$ i.v. 3 times per day, he had significant neurological recovery and was discharged. Although HSE is a relatively rare condition, it is the most common cause of sporadic encephalitis in Western countries. Since the pathogenesis is believed to be due to the reactivation of latent HSV, it is possible that patients who are immunosuppressed are at higher risk for HSE. In addition, patients who are immunosuppressed or immunocompromised often present atypically, which may delay time to diagnosis and treatment, thus significantly worsening prognosis. This case report intends to raise awareness of this severe condition in the context of patients who have received WBRT and immunosuppressive therapy. In addition, important considerations of diagnosis and treatment of HSE in this patient population are discussed.
\end{abstract}


Sermer et al.: Herpes Simplex Encephalitis as a Complication of Whole-Brain Radiotherapy: A Case Report and Review of the Literature

\section{Introduction}

Herpes simplex virus (HSV) is the most common cause of sporadic encephalitis in Western countries, with an incidence of approximately 2-4 cases per million per year [1]. While the pathogenesis of reactivation of latent HSV is not well understood, it is believed to be triggered by several factors such as immunosuppression, trauma, stress, hyperthermia and surgery [2]. The typical presentation of HSV encephalitis (HSE) consists of fever, decreased consciousness and focal neurologic deficits. Clinical presentation, brain MRI and cerebrospinal fluid (CSF) analysis are the cornerstones of diagnosis of HSE. Unilateral temporal lobe involvement is the classic finding seen on imaging. CSF PCR, which has a sensitivity of $98 \%$ and specificity of $94-100 \%$, is considered the gold standard for diagnosis.

It is known that without any treatment, mortality is estimated at around 70\%, and many patients treated with antivirals will suffer from long-term neurological impairments. Early diagnosis and treatment remains the major prognostic indicator [3]. Empiric antiviral therapy with acyclovir should be initiated at the time the diagnosis is suspected, since any delay in antiviral therapy is associated with a less favorable outcome. Raschilas et al. [4] demonstrated that a 2-day delay in treatment from the time of admission is associated with a 3 -fold risk of death or severe neurological disability at 6 months. They also reported significant neurological sequelae in most HSE patients, with only $37 \%$ returning to their baseline conditions.

Atypical presentations present the greatest challenge to early detection and treatment. In immunocompromised patients, for example, there may be negative imaging and absent CSF pleocytosis [5]. It is therefore critical to recognize those individuals who are at higher risk for HSE and to adopt lower thresholds for diagnosis and initiating treatment. This case report presents a patient with stage IV lung adenocarcinoma who underwent whole-brain radiation with dexamethasone therapy and subsequently developed an atypical presentation of HSE.

\section{Case Presentation}

A 55-year-old male with coronary heart disease and heavy tobacco use presented to the Maine Medical Center in June 2013 with several weeks of worsening right-sided neurological symptoms manifested by arm and hand clumsiness, weakness, numbness and facial droop. Brain MRI was positive for multifocal ring-enhancing lesions involving the left posterior frontal lobe, right frontoparietal region and lateral ventricles. CT scan of the chest revealed a large right upper lobe mass with scattered bilateral lung nodules and hilar and mediastinal adenopathy. Biopsy revealed lung adenocarcinoma. He was loaded with dexamethasone 10 mg i.v., which resulted in rapid resolution of his neurological symptoms, and he was discharged in near-baseline condition and able to return to work. Over the next 21 days, he received 14 fractions of 35 Gy of whole-brain radiotherapy (WBRT) with concurrent dexamethasone $12 \mathrm{mg}$ daily. He tolerated treatment well, and he planned on beginning chemotherapy following completion of WBRT.

One week following the last fraction of radiotherapy, he presented again with a 5-day history of progressive fatigue, intermittent fevers of up to $101^{\circ} \mathrm{F}$, confusion, blurred vision and sharp right-sided headache. On admission, he was afebrile, somnolent but oriented and had an otherwise unremarkable neurological exam. A brain MRI demonstrated a marked response to the radiotherapy with almost $80 \%$ reduction of tumor burden. There were no obvious signs of ischemia, inflammation, or new space-occupying lesions. His laboratory 
values were only significant for hyponatremia ( $\mathrm{Na} 129 \mathrm{mEq} / \mathrm{l}$ ) with a urine osmolality of 909 mosm/kg consistent with syndrome of inappropriate antidiuretic hormone secretion (SIADH). Over the next several days, his serum sodium drifted down to $123 \mathrm{mEq} / \mathrm{l}$, which was managed by fluid restriction. On the morning of hospital day 3 , he developed nonconvulsive status epilepticus confirmed by EEG, which showed continuous $2.5-3 \mathrm{~Hz}$ seizure activity in the right hemisphere. After a stat lumbar puncture, he was started empirically on intravenous vancomycin, cefepime and acyclovir for suspected meningoencephalitis. CSF was acellular with an elevated protein level $(70 \mathrm{mg} / \mathrm{dl})$. CSF cell count, cytology, cryptococcal antigen, and paraneoplastic antibodies were negative. Several days later, the HSV-1 PCR results were returned and found to be positive. A repeat brain MRI (hospital day 7) demonstrated marked hyperintensities in the right medial temporal lobe, insular cortex and hippocampal formation most pronounced in T2 (fig. 1) and diffusion-weighted images. Over the next 2 weeks, the patient continued to have daily intermittent fevers and periods of confusion and lethargy, which gradually improved. The patient was kept on acyclovir 10 $\mathrm{mg} / \mathrm{kg}$ i.v. every $8 \mathrm{~h}$ to complete a 21-day course. At discharge on hospital day 25, the patient had residual dysarthria and mild word-finding difficulties but no other neurological deficits.

\section{Discussion and Conclusions}

There is currently no known generally accepted association between WBRT and HSE. In fact, we have counted only 30 documented cases of HSE shortly following WBRT [1, 2, 5-12]. Since immunosuppression is thought to contribute to reactivation of HSV due to breakdown of immune surveillance [6], it is surprising that there does not seem to be a higher incidence of HSE in immunosuppressed or immunocompromised hosts. One explanation is that the clinical features of the encephalitis develop secondary to an exaggerated immune-mediated response that is merely triggered by the virus [13]. In 2011, Graber et al. [1] demonstrated that there was a higher incidence (1:250) of HSE in a cohort of cancer patients treated with prior WBRT and dexamethasone $(n=997)$ at the Memorial Sloan-Kettering compared to the general population (2-4:1 million). It should be recognized, however, that this study is limited by a small sample size and sampling bias (as all are cancer patients and many having received chemotherapy). Chakraborty et al. [7] assert that immunosuppression does in fact promote HSE, and this claim is supported by studies using immunocompromised mouse and human models. Animal models have also demonstrated that corticosteroids promote reactivation of latent HSV [14]. There are several hypotheses that can explain the transposition of HSV into the central nervous system. WBRT is known to cause disruption of the blood-brain barrier. Alternatively, HSV may spread by direct extension from the olfactory mucosa and through the cribriform plate into the orbital frontal or medial temporal lobes [8]. It may also travel to the brain along neuronal axons after reactivation in the trigeminal ganglia.

Despite the low incidence of HSE, clinicians should be vigilant in this group of patients, since early diagnosis and treatment are critical. In their retrospective study of 93 HSE patients, Raschilas et al. [4] found that the average delay in treatment from the time of admission was 2 days and from the reported onset of symptoms 5.5 days. Delayed initiation of acyclovir in most cases can be attributed to low clinical suspicion in the context of either atypical presentation, false negative testing or presence of confounding comorbidities. Poissy et al. [3] reported the following factors associated with delayed treatment: poor premorbid performance status, absence of fever, delay in brain imaging, and CSF white blood cell count $<10 / \mathrm{mm}^{3}$. Delay to treatment may therefore be extended in patients who are 
Sermer et al.: Herpes Simplex Encephalitis as a Complication of Whole-Brain Radiotherapy: A Case Report and Review of the Literature

severely ill due to advanced cancer or immunosuppressed from chemotherapy. Similarly to our patient, all documented cases of HSE following WBRT were notable for absent or minimal CSF cell count in the case series described by Graber et al. [1] as well as Jakob et al. [8]. Moreover, Bloch [14] points out that HSV PCR may even be falsely negative initially in the earlier stages of HSE. CSF may need to be tested serially and the patient continued on acyclovir if clinical suspicion for HSE is high [8]. Furthermore, Koudriavtseva et al. [5] recognized that they initially mistakenly attributed their patient's cognitive impairment to cerebral edema from the WBRT. In our patient, the differential diagnosis of altered mental status also included hyponatremia from SIADH, paraneoplastic encephalitis and progression of brain metastases.

In addition to atypical or even subacute presentations delaying initiation of treatment, immunosuppressed and immunocompromised patients may be at greater risk for refractory or relapsing HSE with more severe manifestations. Increasing antiviral drug resistance of HSV-1 has been observed in immunocompromised patients. The incidence of acyclovir resistance is estimated to be $0.5 \%$ in immunocompetent individuals but may be up to $14 \%$ in immunocompromised individuals [15]. This raises the possibility that individuals who appear to be refractory to traditional therapy with acyclovir may not have been incorrectly diagnosed but in fact are infected with a resistant strain of HSV-1. In such cases, alternative antivirals like foscarnet or cidofovir may be considered [5]. However, it is important to note that the effectiveness of antivirals may be limited in immunosuppressed patients as they can only inhibit viral replication but require the host immune system to suppress or fully eradicate the virus [6]. Of note, Lizarraga et al. [10] presented a case where HSE was successfully treated by the addition of $40 \mathrm{mg}$ i.v. dexamethasone to the patient's acyclovir regimen. They propose that corticosteroids can decrease the exaggerated inflammatory response seen in HSE, much like in other similar processes such as bacterial meningitis or herpetic keratitis.

Our patient was not known to have a past history of HSV infection. Due to the widespread prevalence of latent HSV and the low incidence of HSE, HSV screening is probably not warranted in all patients prior to receiving cranial irradiation. However, given the potential serious consequences of HSE, HSV serologies could be considered for those patients with reported histories of recurrent HSV episodes [2]. While the results should not change the management of the patient's brain metastases, positive serologies should merit closer observation for HSE symptoms. Additionally, one could consider prophylactic oral acyclovir or valacyclovir concurrent with radiation and potentially for an extended period following completion of therapy [9]. 
Sermer et al.: Herpes Simplex Encephalitis as a Complication of Whole-Brain Radiotherapy: A Case Report and Review of the Literature

\section{References}

1 Graber JJ, Rosenblum MK, DeAngelis LM: Herpes simplex encephalitis in patients with cancer. J Neurooncol 2011;105:415-421.

-2 Silvano G, Lazzari G, Resta F, et al: A herpes simplex virus-1 fatal encephalitis following chemoradiotherapy, steroids, and prophylactic cranial irradiation in a small cell lung cancer patient. Lung Cancer 2007;57:243-246.

-3 Poissy J, Wolff M, Dewilde A, et al: Factors associated with delay to acyclovir administration in 184 patients with herpes simplex virus encephalitis. Clin Microbiol Infect 2009;15:560-564.

4 Raschilas F, Wolff M, Delatour F, et al: Outcome of and prognostic factors for herpes simplex encephalitis in adult patients: results of a multicenter study. Clin Infect Dis 2002;35:254-260.

-5 Koudriavtseva T, Onesti E, Tonachella R, et al: Fatal herpetic encephalitis during brain radiotherapy in a cerebral metastasized breast cancer patient. J Neurooncol 2010;100:137-140.

-6 Tan IL, McArthur JC, Venkatesan A, Nath A: Atypical manifestations and poor outcome of herpes simplex encephalitis in the immunocompromised. Neurology 2012;79:2125-2132.

7 Chakraborty C, Donner M, Colan D: Fatal herpes encephalitis in a patient with small cell lung cancer following prophylactic cranial radiation - a case report with review of literature. Anticancer Res 2013;33:3263-3268.

8 Jakob NJ, Lenhard T, Schnitzler P, et al: Herpes simplex virus encephalitis despite normal cell count in cerebrospinal fluid. Crit Care Med 2012;40:1304-1308.

-9 Okada M, Miyake K, Shinomiya A, et al: Relapse of herpes encephalitis induced by temozolomide-based chemoradiation in a patient with malignant glioma. J Neurosurg 2013;118:258-263.

-10 Lizarraga K, Alexandre LC, Ramos-Estebanez C, Merenda A: Are steroids a beneficial adjunctive therapy in the immunosuppressed patient with herpes simplex virus encephalitis? Case Rep Neurol 2013;5:52-55.

-11 Matikas A, Kontopodi E, Nintos G, et al: A case of herpes simplex-associated encephalitis after brain irradiation for lung cancer metastases. Anticancer Res 2014;34:4411-4414.

12 Lins H, Kanakis D, Heinrichs T, et al: Sudden progression of a glioblastoma in partial remission? Clin Neurol Neurosurg 2004;106:335-336.

13 Hudson SJ, Dix RD, Streilein JW: Induction of encephalitis in SJL mice by intranasal infection with herpes simplex virus type 1: a possible model of herpes simplex encephalitis in humans. J Infect Dis 1991;163:720.

14 Bloch KC: Atypical herpes simplex encephalitis after total cranial irradiation: casting a wider net? Crit Care Med 2012;40:1385-1386.

15 Stranska R, Schuurman R, Nienhuis E, et al: Survey of acyclovir-resistant herpes simplex virus in the Netherlands: prevalence and characterization. J Clin Virol 2005;32:7-18. 


\section{Case Reports in Oncology}
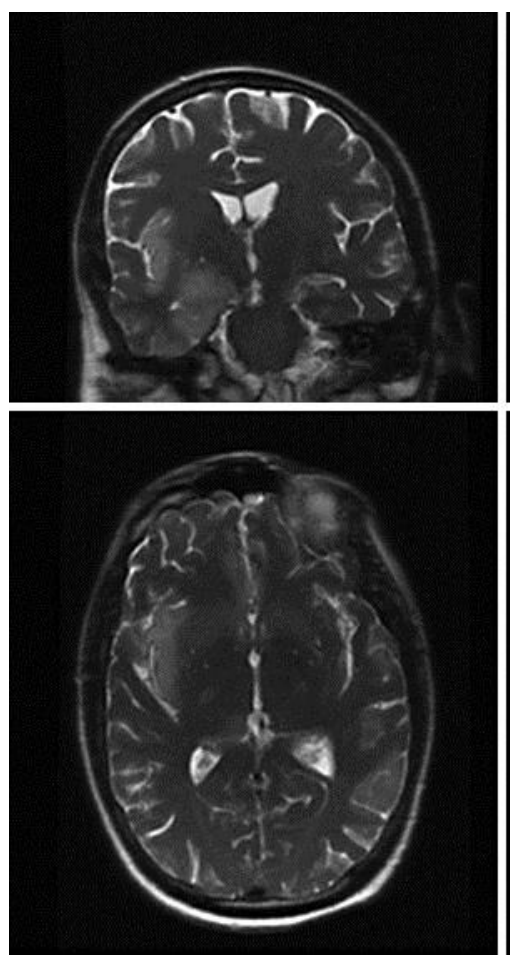

\begin{tabular}{l|l}
\hline Case Rep Oncol 2014;7:774-779 \\
\hline DOI: 10.1159/000369527 & $\begin{array}{l}\text { C 2014 S. Karger AG, Basel } \\
\text { www.karger.com/cro }\end{array}$ \\
\hline
\end{tabular}

Sermer et al.: Herpes Simplex Encephalitis as a Complication of Whole-Brain Radiotherapy: A Case Report and Review of the Literature
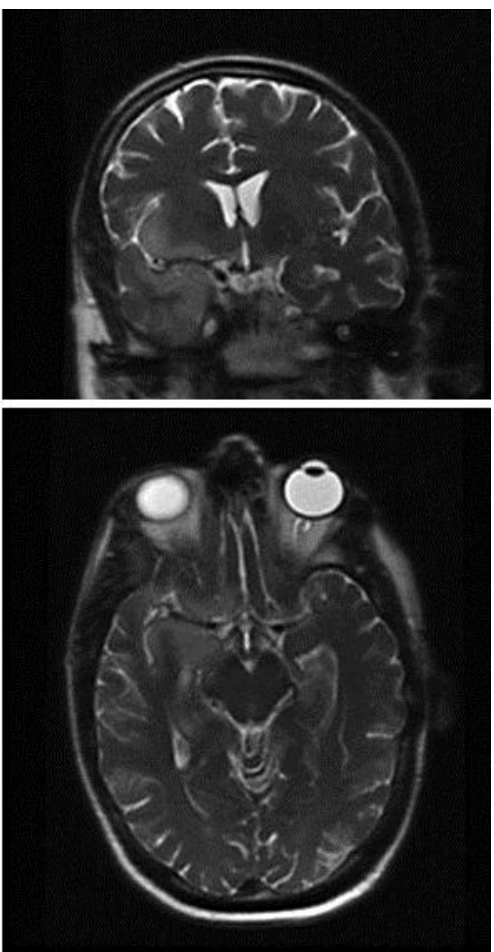

Fig. 1. Coronal (top) and axial (bottom) T2-weighted MRI of the brain on hospital day 7 demonstrating marked T2 prolongation and gyriform swelling of the right mesial temporal lobe, insular cortex and hippocampal formation. These findings are new compared to prior imaging from the day of admission. 